\section{Rektalabstrich vor Biopsie deckt Resistenzen auf}

\author{
Ein frühzeitiger Rektalabstrich vor einer transrektalen Prostatabiopsie liefert \\ verlässliche Informationen über das bakterielle Spektrum im Bereich der \\ Entnahmestelle und erlaubt somit eine zielgerichtete Antibiotikaprophylaxe.
}

$D^{i}$ e transrektale ultraschallgesteuerte Prostatabiopsie (TRUS) birgt ein gewisses Infektionsrisiko. Um Komplikationen wie Infektionen zu vermeiden, führt man daher routinemäßig eine Antibiotikaprophylaxe durch. Das Timing dieser Prophylaxe stellt den Arzt vor ein Dilemma: Erfolgt diese erst kurz vor dem Eingriff, ist eine zielgerichtete Therapie, die das Anlegen einer Bakterienkultur erfordert, nicht möglich. Andererseits läuft man bei zu reichlich bemessenem Zeitabstand Gefahr, dass sich das Keim- und Resistenzspektrum verändert und es zu falschen Ergebnissen kommt.

In der vorliegenden Studie konnte gezeigt werden, dass ein etwa zwei Wochen vor der Biopsie vorgenommener Rektalabstrich verlässliche Ergebnisse im Hin- blick auf Fluorchinolon-Resistenzen (FQR) liefert. Die Ergebnisse stimmten zu $93 \%$ mit einem Kontrollabstrich unmittelbar vor der Biopsie überein. Die Forscher hatten 108 Biopsiekandidaten rektale Abstriche entnommen und Kulturen angelegt. Ausgewertet wurden 58 Patienten, bei denen bakterielles Wachstum (E. coli) nachweisbar war und von denen man unmittelbar vor der Biopsie, im Schnitt nach zwei Wochen, erneut einen Abstrich gewinnen konnte. Alle Patienten erhielten - beginnend mit dem Morgen der Biopsie - über drei Tage Ciprofloxacin $500 \mathrm{mg}$ alle zwölf Stunden. In den 30 Tagen nach der Biopsie entwickelte sich bei keinem der Teilnehmer eine Infektion. Insgesamt waren die Ergebnisse der Abstriche bei 54 Kultur-Paaren konkor- dant. Eine Diskordanz zwischen erstem und zweitem Ergebnis lag bei vier Paaren vor. Bei zwei Patienten war jeweils das erste Ergebnis falsch positiv, bei zwei weiteren hatten sich Pseudomonas spp. gebildet. Die Sensitivität des Screenings lag bei $95,5 \%$, die Spezifität bei $77,8 \%$.

Fazit: Den FQ-R-Status zu bestimmen hat nicht nur den Vorteil, über eine zielgerichtete Antibiotikatherapie eine Infektion verhindern zu können, damit lasse sich auch ein nutzloser Einsatz von Antibiotika reduzieren, so die Autoren. Zudem fiel in der Studie eine eindeutige Lernkurve bei der Qualität der Abstriche auf. Bei der Abstrichentnahme solle man daher darauf achten, dass man auch wirklich Stuhl „erwischt“. Ein Kontrollagar ohne Antibiotikazusatz sei in jedem Falle hilfreich. Dr. Elke Oberhofer

Liss MA et al. Screening Rectal Culture to Identify Fluoroquinolone-resistant Organisms Before Transrectal Prostate Biopsy: Do the Culture Results Between Office Visit and Biopsy Correlate? Urology 2013; 82: 67-73

\title{
Weniger PSA-Tests in den Niederlanden
}

\section{Die Empfehlung gegen ein allgemeines PSA-basiertes Prostatakrebs- Screening hat in den Niederlanden nicht nur die gewünschte Wirkung gezeigt: Auch indizierte Kontrollen wurden in der Folge häufiger unterlassen.}

D urch ein PSA-basiertes Screening bei Männern zwischen 50 und 74 kann die Prostatakrebs-Mortalität um $20 \%$ gesenkt werden. Die absolute Risikoreduktion ist jedoch gering und wird mit einer hohen Quote von Überdiagnosen und Übertherapien erkauft: Um einen krebsspezifischen Todesfall $\mathrm{zu}$ verhindern, müssen 1.410 Männer zweimal alle vier Jahre getestet werden und 48 Prostatakarzinome werden unnötigerweise behandelt - so das Ergebnis der im Jahr 2009 veröffentlichten ERSPC-Studie [Schröder FH et al. N Engl J Med 2009; 360: 1320-28]. Die Studie wurde kurz danach zusammen mit einem Kommentar des nationalen HausärzteVerbandes an alle niederländischen Hausärzte versendet. Wegen des ungünstigen Nutzen-Risiko-Verhältnisses sprach sich der Verband eindeutig gegen ein Screening aus. Wie sich diese Empfehlung auf das PSA-Testungs-Verhalten der Hausärz- te ausgewirkt hat, wurde nun in einer Studie untersucht. Die Mediziner werteten dazu Daten einer Krankenversicherung und eines Großlabors aus dem Jahr vor und dem Jahr nach der ERSPC-Studie aus.

Die Krankenkasse hatte in dieser Zeit mehr als 120.000 PSA-Tests erstattet (in den Niederlanden werden alle PSA-Tests ersetzt), gut die Hälfte waren Erstuntersuchungen. Circa vier Monate nach der ERSPC-Publikation ging die Testhäufigkeit vor allem bei Männern ab 60 zurück. Insgesamt sank die Testquote von 33,09 auf 27,52 pro 1.000 Männer und Jahr.

Auch die Rate der Folgetests innerhalb eines Jahres ging nach ERSPC zurück, den Labordaten zufolge von vorher 38,5\% auf dann $26,5 \%$. Bei unauffälligen PSA-Werten $(<4 \mathrm{ng} / \mathrm{ml})$ war sogar eine Reduktion von $31,8 \%$ auf $18,8 \%$ festzustellen. Allerdings gab es auch dann weniger Nachkontrollen, wenn der PSA-Wert beim ersten
Test mäßig erhöht gewesen war (4-10 ng/ $\mathrm{ml})$, statt in $65,3 \%$ nur noch in $52,2 \%$ der Fälle. Patienten mit auffälligen PSA-Werten $(>4 \mathrm{ng} / \mathrm{ml})$ wurden nach der ERSPCPublikation nur noch zu 23,5\% zur Abklärung an einen Urologen überwiesen, vorher waren es $28,3 \%$ gewesen.

Fazit: Die Nachkontrollen nach auffälligen PSA-Werten seien schon vor der ERSPC-Publikation nicht adäquat gewesen und danach erst recht nicht, kritisieren die Autoren. Eventuell hätten die Hausärzte die Empfehlung ihres Verbandes, PSA-Tests einzuschränken, falsch interpretiert. Andererseits wurde auch bei Männern unter 40 und sogar über $80 \mathrm{Jah}$ ren der PSA-Spiegel bestimmt. Hier seien die ERSPC-Daten, die sich ja auf 50- bis 74-Jährige beschränken, möglicherweise dahingehend fehlgedeutet worden, dass ein genereller Nutzen des PSA-Screenings belegt sei. Dr. Beate Schumacher

van der Meer S et al. Impact of the European Randomized Study of Screening for Prostate Cancer (ERSPC) on prostate-specific antigen (PSA) testing by Dutch general practitioners. BJU Int 2013; 112: 26-31 